\title{
SOME EXAMPLES OF RELATIONS BETWEEN NON-STABLE INTEGRAL COHOMOLOGY OPERATIONS
}

\author{
ANDREW PERCY
}

\begin{abstract}
The algebraic structure of the natural integral cohomology operations is explored by means of examples. We decompose the generators of the groups $H^{m}(\mathbb{Z}, n)$ with $2 \leq n \leq 7$ and $2 \leq m \leq 13$ into the operations of cup products, cross-cap products and compositions. Examination of these decompositions and comparison with other possible generators demonstrates the existence of relations between integral operations that have withheld formulation. The calculated groups and generators are collected in a table for practical reference.
\end{abstract}

\section{Introduction}

$H^{m}(\mathbb{Z}, n)$ is the $m$-th integral cohomology group of the Eilenberg-Mac Lane space $K(\mathbb{Z}, n)$, elements of which may be considered as maps $K(\mathbb{Z}, n) \rightarrow$ $K(\mathbb{Z}, m)$. For $X$ a connected $C W$-complex, when considering $n$-ary natural cohomology operations $H^{m_{1}}(X) \times \cdots \times H^{m_{n}}(X) \rightarrow H^{p}(X)$, the universal examples of these operations are given by elements of the group

$$
H^{p}\left(K\left(\mathbb{Z}, m_{1}\right) \times \cdots \times K\left(\mathbb{Z}, m_{n}\right)\right) .
$$

The Künneth formula shows that all such operations can be generated (under composition of universal examples) by cup products, cross-cap products and composition operations [7]. The algebraic object $H^{*}(X)$ consisting of the graded cohomology groups of $X$ together with all $n$-ary natural cohomology operations has not been explicitly determined. These objects, which we may call coП-algebras since they are Eckmann-Hilton dual to the П-algebras of homotopy theory, can (dual to $\Pi$-algebras) be described implicitly as a functor from the category of finite products of integral Eilenberg-Mac Lane spaces to the category of pointed sets [7]. Unlike the ח-algebras (see [2]) there is no explicit description of the coП-algebras because, although a set of generators of the operations are known ${ }^{1}$, not all of the relations on these generators have

Received July 25, 2008; Revised May 13, 2009.

2000 Mathematics Subject Classification. 55S05.

Key words and phrases. integral cohomology operation, relations.

${ }^{1}$ Compositions, cup products and cross-cap products are not the only possible set of generating operations. 
explicit formulations. In this article we determine the generators of a range of $H^{m}(\mathbb{Z}, n)$, enabling us to demonstrate relations between the generating operations.

It was shown in [7] that the coI-algebras of the integral Eilenberg-Mac Lane spaces are free objects in the sense that the cohomology group $H^{m}(\mathbb{Z}, n)$ is freely generated by all combinations of operations with image in that group, subject only to the relations on those operations. It follows that if we have a decomposition of the generators of each summand of $H^{m}(\mathbb{Z}, n)$, then any composition of operations that should generate a summand in $H^{m}(\mathbb{Z}, n)$ must be related (perhaps trivially) to one of the decompositions already determined. Thus, by determining a decomposition of each summand in $H^{m}(\mathbb{Z}, n)$ into cup products, cross-cap products and composition operations we can then determine the existence, in some instances, of relations with other natural operations expected to be found in $H^{m}(\mathbb{Z}, n)$. The generator of a summand in $H^{m}(\mathbb{Z}, n)$ which does not decompose into cup products, cross-cap products and composition operations is called indecomposable and is itself a composition operation.

There are several methods, apart from the one presented here, for determining the groups $H^{m}(\mathbb{Z}, n)$, however these methods do not provide a decomposition of the generators of the group into the three natural operations generating all others. The earliest significant calculations of a small range of groups were made by Eilenberg and Mac Lane [3]. Cartan [1] uses the method of 'constructions' to fully determine the cohomology algebra of $H^{*}(\mathbb{Z}, n ; \mathbb{Z} / p)$ for prime $p$ and also $H_{*}(\mathbb{Z}, n)$, however the method falls short of calculating $H^{*}(\mathbb{Z}, n)$. Nevertheless, the calculations of $H_{*}(\mathbb{Z}, n)$ together with the Universal Coefficient Theorem can be used to calculate the groups $H^{m}(\mathbb{Z}, n)$, although the lifting of the homology operation structure to cohomology has only been partially determined by Kochman [4] for stable compositions. Kochman gives a means of calculating $H^{*}(X)$ as a module over the stable compositions for spaces $X$ which have only simple torsion in their homology groups (such as Eilenberg-MacLane spaces). May's results in [5], §10, involving the Bockstein spectral sequence applied over a range of modulo $p$ cohomologies provide another calculation of the groups $H^{m}(\mathbb{Z}, n)$ as having a summand of $\mathbb{Z} / p$ whenever $\beta_{1}: H^{m}(\mathbb{Z}, n ; \mathbb{Z} / p) \rightarrow H^{m+1}(\mathbb{Z}, n ; \mathbb{Z} / p)$ is non-trivial and a summand of $\mathbb{Z}$ for each element persisting to $E_{\infty}$, but again, without determining the algebraic structure of the integral operations overlaid on these groups. The possibility of coherently pulling back the integral coП-algebra from the cohomology modulo $p$ algebras is not the direction of research followed in this article, however, the results shown in Table 1 when localized and compared with the generators of the groups $H^{m}(\mathbb{Z}, n ; \mathbb{Z} / p)$ given by Cartan [1] provide interesting examples of this possibility. The initial study in this direction has indicated the need to determine the distributive properties of Bocksteins over composition and cup products which may not yet be known (they were not known to Steenrod [8]).

In this study, we use the Leray-Serre spectral sequence for the path-loop fibration $K(\mathbb{Z}, n) \rightarrow P K(\mathbb{Z}, n+1) \rightarrow K(\mathbb{Z}, n+1)$ to calculate the groups 
$H^{m}(\mathbb{Z}, n)$ for $2 \leq n \leq 7$ and $2 \leq m \leq 13$. These are given in Table 1 . The groups $H^{m}(\mathbb{Z}, n)$, with a chosen decomposition of their generators, do not appear to have been compiled into a table for practical reference as was done for the Eckmann-Hilton dual objects of primary homotopy operations [9]. The advantage to using the Leray-Serre spectral sequence is its simplicity and explicit description of the cup product structure of the primary integral operations. The initial results of the calculation can be reused to remove the uncertainty regarding generators of $H^{m}(\mathbb{Z}, n)$ which occurs when the decomposition of a group generator is not determined by the algebraic structure of cup product inherent to the spectral sequence. The known relations and the stable composition module structure of [4] can also be applied to rule out possible generators (see Section 4). Examination of the generators and comparison to undetected decompositions of these generators leads to some examples of, as yet, unformulated relations (see Section 5).

The spectral sequence is set up (see Appendix A) simply as a tensor product of graded algebras converging to 0 for the range of $H^{m}(\mathbb{Z}, n)$ calculated and we give a sample calculation for $H^{*}(\mathbb{Z}, 3$ ) (see Appendix B). Using known modulo $p$ information to assist in the calculation of the differentials, the current method could be used to give further results to those reported in Table 1.

\section{A table of integral cohomology operations}

Table 1 gives the results for the unstable cohomology groups $H^{m}(\mathbb{Z}, n)$ together with a chosen generator for $H^{n}(\mathbb{Z}, n)$. This chosen generator is just the identity map $K(\mathbb{Z}, n) \rightarrow K(\mathbb{Z}, n)$. Generators are denoted by angle brackets and product notation is used for cup product so that, for example, $b c=b \cup c$ and $b^{2}=b \cup b$. The transgression $\Omega^{-1}$ is the inverse of the loop functor. The stable stems are shown but the stable groups omitted.

\section{Known relations on integral operations}

If we consider all natural cohomology operations to be generated by compositions, cup products and cross-cap products, then it only remains to determine all relations between these generating operations to have an explicitly described cohomology algebra. Of course, there is also the group addition operation within the cohomology groups so relations with this addition must also be determined.

There are only two relations between integral operations known to the author apart from the bilinearity and graded commutativity of cup product and the additivity of primitive (including stable) compositions. If we have operations $x, y$ and $z$, in groups for which the following relations are defined, then

$$
\begin{aligned}
& (x+y) \circ z=(x \circ z)+(y \circ z) \text { and } \\
& (x \cup y) \circ z=(x \circ z) \cup(y \circ z) .
\end{aligned}
$$


TABLE 1 . The groups $H^{m}(\mathbb{Z}, n)$

\begin{tabular}{|c|c|c|c|c|c|c|c|}
\hline$m$ & & & & & & & \\
\hline 13 & 0 & $\mathbb{Z} / 2\langle b d\rangle$ & $\begin{array}{c}\mathbb{Z} / 5\langle j\rangle \\
\oplus \\
\mathbb{Z} / 3\langle f h\rangle\end{array}$ & $\mathbb{Z} / 2\left\langle k \Omega^{-1} g\right\rangle$ & $\mathbb{Z} / 2\langle q\rangle$ & 0 & \\
\hline 12 & $\mathbb{Z}\left\langle a^{6}\right\rangle$ & $\begin{array}{c}\mathbb{Z} / 2\left\langle b^{4}\right\rangle \\
\oplus \\
\mathbb{Z} / 5\langle e\rangle \\
\end{array}$ & $\mathbb{Z}\left\langle f^{3}\right\rangle$ & $\mathbb{Z} / 2\langle m\rangle$ & $\mathbb{Z}\left\langle n^{2}\right\rangle$ & & \\
\hline 11 & 0 & $\mathbb{Z} / 3\langle b c\rangle$ & $\begin{array}{c}\mathbb{Z} / 2\langle f g\rangle \\
\oplus \\
\mathbb{Z} / 2\langle i\rangle\end{array}$ & 0 & $\begin{array}{c}\mathbb{Z} / 2\langle o\rangle \\
\oplus \\
\mathbb{Z} / 3\langle p\rangle\end{array}$ & & \\
\hline 10 & $\mathbb{Z}\left\langle a^{5}\right\rangle$ & $\mathbb{Z} / 2\langle d\rangle$ & 0 & $\begin{array}{c}\mathbb{Z} / 2\left\langle k^{2}\right\rangle \\
\oplus \\
\mathbb{Z} / 3\langle l\rangle\end{array}$ & & & \\
\hline 9 & 0 & $\mathbb{Z} / 2\left\langle b^{3}\right\rangle$ & $\mathbb{Z} / 3\langle h\rangle$ & 0 & & & \\
\hline 8 & $\mathbb{Z}\left\langle a^{4}\right\rangle$ & $\mathbb{Z} / 3\langle c\rangle$ & $\mathbb{Z}\left\langle f^{2}\right\rangle$ & & & & \\
\hline 7 & 0 & 0 & $\mathbb{Z} / 2\langle g\rangle$ & & & $\langle r\rangle$ & \\
\hline 6 & $\mathbb{Z}\left\langle a^{3}\right\rangle$ & $\mathbb{Z} / 2\left\langle b^{2}\right\rangle$ & & & $\langle n\rangle$ & & \\
\hline 5 & 0 & 0 & & $\langle k\rangle$ & & & \\
\hline 4 & $\mathbb{Z}\left\langle a^{2}\right\rangle$ & & $\langle f\rangle$ & & & & \\
\hline 3 & 0 & $\langle b\rangle$ & & & & & \\
\hline 2 & $\mathbb{Z}\langle a\rangle$ & & & & & & \\
\hline & 2 & 3 & 4 & 5 & 6 & 7 & $n$ \\
\hline
\end{tabular}

These are easily shown by considering the groups $H^{m}(\mathbb{Z}, n)$ to be homotopy classes of maps $K(\mathbb{Z}, n) \rightarrow K(\mathbb{Z}, m)$. For instance, if $h_{\cup} \in H^{p+q}(K(\mathbb{Z}, p) \times$ $K(\mathbb{Z}, q))$ is the universal example for cup product, then the equation (2) is given by the diagram

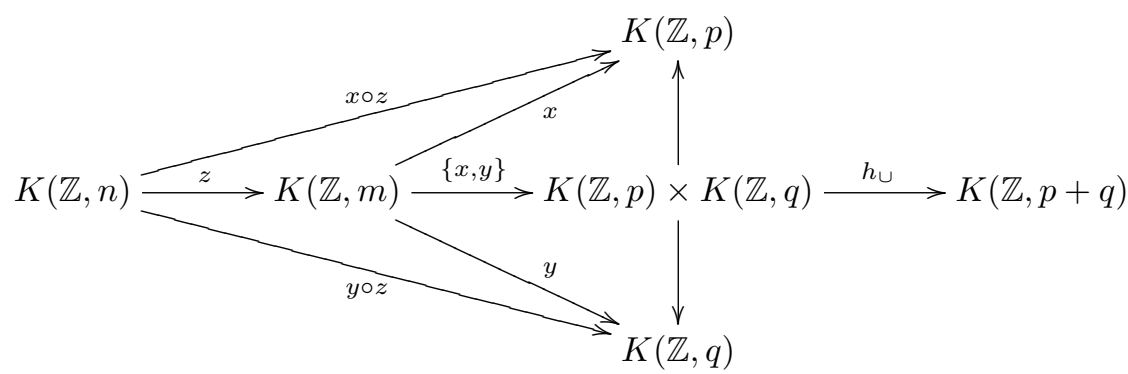

where $\{x, y\}$ is the canonical map into the product. We have examples of this relation at work. For instance, recalling that $f$ is the identity map,

$$
f^{2} \circ a^{2}=(f \cup f) \circ a^{2}=\left(f \circ a^{2}\right) \cup\left(f \circ a^{2}\right)=a^{2} \cup a^{2}=a^{4} .
$$


We also see that $n^{2} \circ b^{2}=b^{4}$ for the same reasons and clearly $x^{2} \circ y=y^{2}$ for $x$ any generator of $H^{n}(\mathbb{Z}, n)$.

Compositions, in general, may be unstable (in fact, non-primitive), so the expression $z \circ(x+y)$ does not have a known decomposition. We will also look at examples of the expression $z \circ(x \cup y)$.

We will show several cases where we know relations are at work and they cannot be explained by the known relations (1) and (2) above. The set of all primary cohomology operations are in bijection with their universal examples; the maps in the homotopy category of products of Eilenberg-Mac Lane spaces. Some of these maps are related, such as $h_{\cup} \circ\{x, y\} \circ z$ and $h_{\cup} \circ\{x \circ z, y \circ z\}$ in the diagram above. If any composition of maps is a universal example for an operation acting within the coП-algebra $H^{*}(\mathbb{Z}, n)$, and its image is a non-trivial group, then there must be some relation forcing this map to be equivalent to some multiple (perhaps a trivial multiple) of the generator we have chosen for that non-trivial summand.

The properties of cross-cap products in cohomology have not been given beyond the definition [7], though the homology version of these products has been used to advantage in the calculation of the homology of Eilenberg-Mac Lane spaces [3]. These products exist when $x \in H^{m}(\mathbb{Z}, n) \cong \mathbb{Z} / s$ and $y \in$ $H^{p}(\mathbb{Z}, q) \cong \mathbb{Z} / t$ and give an element $x \overline{\times} y \in H^{m+p-1}(K(\mathbb{Z}, n) \times K(\mathbb{Z}, q))$ in the summand $\mathbb{Z} / \operatorname{gcd}(s, t)$ that is given by the Tor summand of the Künneth formula.

\section{Determining generators}

Not all of the generators shown in Table 1 were given directly from the spectral sequence. Any generator that is decomposable as a cup product is given by the algebraic structure of the spectral sequence, however groups calculated on later pages of the spectral sequence are not shown to be decomposable without further investigation. For instance, the generator $d$ of $H^{10}(\mathbb{Z}, 3)$ could be indecomposable or one of the compositions $x \circ b^{2}, y \circ c$ or $z \circ b^{3}$ with $x \in H^{10}(\mathbb{Z}, 6)$, $y \in H^{10}(\mathbb{Z}, 8)$ and $z \in H^{10}(\mathbb{Z}, 9)$. Reusing the later calculations given in Table 1 and stability, each of the groups containing $x, y$ and $z$ are trivial so we can conclude that $d$ is indecomposable. There can be no cross-cap products for dimensional reasons.

In Table $1, H^{12}(\mathbb{Z}, 3)$ has a summand of $\mathbb{Z} / 5$ generated by $e$. Looking at the possible compositions and ruling out those that compose with an element of a trivial group, we arrive at the possibilities that $e$ is indecomposable or generated by $x \circ b^{2}$ or $y \circ b^{3}$ with $x \in H^{12}(\mathbb{Z}, 6) \cong \mathbb{Z}$ and $y \in H^{12}(\mathbb{Z}, 9) \cong \mathbb{Z} / 2$. However, using relation (1), since $y$ has order 2 , we get

$$
0=0 \circ b^{3}=(y+y) \circ b^{3}=\left(y \circ b^{3}\right)+\left(y \circ b^{3}\right)=2\left(y \circ b^{3}\right)
$$

so that $y \circ b^{3}$ has order 2 hence could not generate the summand $\mathbb{Z} / 5$.

With $x \in H^{12}\left(K^{6}\right) \cong \mathbb{Z}\left\langle n^{2}\right\rangle$ we have $x=\alpha n^{2}$ for $\alpha \in \mathbb{Z}$. Then using the relation $n^{2} \circ b^{2}=b^{4}$ as well as (1) we get $\left(\alpha n^{2}\right) \circ b^{2}=\alpha\left(n^{2}\right) \circ b^{2}=\alpha b^{4}$ 
so that $x \circ b^{2}$ is not in the $\mathbb{Z} / 5$ summand but, rather, we have the relation equating $x \circ b^{2}$ with the generator of the $\mathbb{Z} / 2$ summand. Thus, we see that $e$ is indecomposable.

For $H^{7}(\mathbb{Z}, 4)$ and $H^{9}(\mathbb{Z}, 4)$ the only possible compositions are with trivial groups so we know that $g$ and $h$ are indecomposable. The same argument applies to show that $l$, generating a summand of $H^{10}(\mathbb{Z}, 5)$, and the generators $o, p$ of the summands of $H^{11}(\mathbb{Z}, 6)$ are also indecomposable. There can be no cross-cap products for dimensional reasons.

The generator, $i$, of the second $\mathbb{Z} / 2$ summand of $H^{11}(\mathbb{Z}, 4)$ could be indecomposable or a stable composition $\Omega^{-4} g \circ f^{2}$, with $\Omega^{-4} g \in H^{11}(\mathbb{Z}, 8)$ being the only non-trivial element of $H^{11}(\mathbb{Z}, 8)$. Kochman [4] has determined the action of the stable compositions on the cohomology groups of a space for a large class of spaces including Eilenberg-Mac Lane spaces. Corollary 4.15 of [4] pairs a coaction on the homology groups to stable compositions on the cohomology groups of this class of spaces. Theorem 4.8 of [4] gives the coaction paired with $\Omega^{-4} g \circ f^{2}$ as $\Delta^{\prime \prime}: \widetilde{H}_{11}(\mathbb{Z}, 4) \rightarrow \sum_{s+t=10} H_{s}(\mathbb{Z}, 8) \otimes H_{t}(\mathbb{Z}, 4)$. This is a trivial coaction since $H_{s}(\mathbb{Z}, 8)=0$ for $1 \leq s \leq 7, H_{t}(\mathbb{Z}, 4)=0$ for $1 \leq t \leq 3$ and, by [4] Theorem 4.8(b), $\Delta^{\prime \prime}$ is reduced. Consequently, $\Omega^{-4} g \circ f^{2}$ is trivial and $i$ is indecomposable.

All compositions that could generate the $\mathbb{Z} / 5$ summand of $H^{13}(\mathbb{Z}, 4)$ are either with elements of trivial groups or, in the case of $x \circ f^{2}$ with $x \in H^{13}(\mathbb{Z}, 8)$, of the wrong torsion to generate $\mathbb{Z} / 5$. Then, the generator of the $\mathbb{Z} / 5$ summand of $H^{13}(\mathbb{Z}, 4)$ is undetermined, by the spectral sequence, to be either indecomposable or a cross-cap product $(g \overline{\times} g) \circ\{f, f\}$ shown in the diagram.

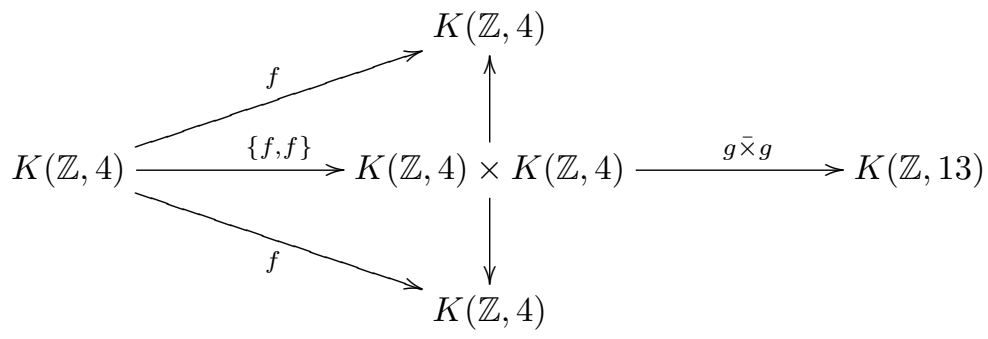

However, $g \overline{\times} g$ has order 2 since the greatest common divisor $\operatorname{gcd}(2,2)=2$, and hence

$$
0=0 \circ\{f, f\}=(g \overline{\times} g+g \overline{\times} g) \circ\{f, f\}=2((g \overline{\times} g) \circ\{f, f\}) .
$$

Thus, $(g \overline{\times} g) \circ\{f, f\}$ has order 2 and hence cannot generate this summand which is therefore indecomposable and denoted $j$. This demonstrates a trivial relation involving cross-caps.

The groups $H^{12}(\mathbb{Z}, 5)$ and $H^{13}(\mathbb{Z}, 6)$ cannot decompose as compositions or cup products since all relevant decompositions are with elements of trivial groups. The only possible cross-cap products involve the $\operatorname{Tor}\left(H^{i}(\mathbb{Z}, n), H^{j}(\mathbb{Z}, m)\right)$ 
group with $H^{i}(\mathbb{Z}, n)$ either 0 or free so must be trivial. Hence, these groups are generated by indecomposable elements $m$ and $q$.

\section{Interesting observations}

We saw that $0=(g \overline{\times} g) \circ\{f, f\} \in H^{13}(\mathbb{Z}, 4)$ was a trivial relation that concerns cross-cap products. The cross cap product $b^{2} \overline{\times} b^{2} \in H^{11}(K(\mathbb{Z}, 3) \times$ $K(\mathbb{Z}, 3))$ should generate a summand $\left(b^{2} \overline{\times} b^{2}\right) \circ\{b, b\} \in H^{11}(\mathbb{Z}, 3)$. However, since $b^{2}$ has order $2, b^{2} \overline{\times} b^{2}$ also has order 2 as does $\left(b^{2} \overline{\times} b^{2}\right) \circ\{b, b\}$ (by relation (1)) which therefore must be trivial in $H^{11}(\mathbb{Z}, 3) \cong \mathbb{Z} / 3\langle b c\rangle$. Thus we have another trivial relation $\left(b^{2} \overline{\times} b^{2}\right) \circ\{b, b\}=0(b c)$.

Trivial in the group $H^{11}(\mathbb{Z}, 3)$ or related to the generator $b c$, are the compositions $o \circ b^{2}$ and $p \circ b^{2}$. Whereas, when applying relation (1) to $0=(o+o) \circ b^{2}$ we see $o \circ b^{2}=0$, when applying relation (1) to $0=(p+p+p) \circ b^{2}$ we could get $p \circ b^{2}=0$, or $b c$, or $2 b c$. A decomposition for left distributivity of composition over cup product (if one exists) would enable us to decide whether or not these operations are trivial. Such a decomposition would be the analogue, for non-primitive operations and integer coefficients, of the Cartan formula for stable operations over $\mathbb{Z} / p$, so there is reason to suppose that it does exist.

There are relations using stability exhibited in the table. $H^{13}(\mathbb{Z}, 3) \cong \mathbb{Z} / 2$ is generated by $b d$ but there should also be an element $x \circ c$, with $x \in H^{13}(\mathbb{Z}, 8)$, in this group. However $H^{13}(\mathbb{Z}, 8)$ is in the stable range so $x$ is $\Omega^{-2} o$ or $\Omega^{-2} p$, where $\Omega^{-2}$ denotes an iterated transgression. The additivity of the stable composition with $\Omega^{-2} o$ tells us that $0=\Omega^{-2} \circ \circ 0=\Omega^{-2} \circ \circ(c+c+c)=3\left(\Omega^{-2} o \circ c\right)$ and hence $\Omega^{-2} o \circ c=0$ so this element is trivially related to $b d$ and similarly for $\Omega^{-2} p$. Interestingly, we see that there is not necessarily a unique stable composition operation for integral operations unlike the case for cohomology over the finite fields.

The integral operations show other features not seen amongst the modulo $p$ algebras. We show in Appendix B, that $b \cup b \cup c$ is trivial in $H^{14}(\mathbb{Z}, 3)$ so some relation is forcing it to be so. Interestingly, this shows that the algebra structure over cup product is not freely generated on all indecomposable elements as is the case for the modulo $p$ algebras calculated by Cartan using 'constructions' [1].

Presumably, the place to look for the entirety of relations would be at a cochain complex level, since all three types of primary operation can be defined there. Cross-cap products, at this stage, are only defined through the fact that if a cocycle $\beta \in C^{n+1}$ has $s$-torsion, then there is a coboundary $\alpha \in C^{n}$ with $\partial(\alpha)=s \beta$ with the coboundary $\alpha$ not necessarily appearing as an element in any cohomology group. Some specific relations such as a decomposition of $z \circ(x \cup y)$ may be open to many different approaches but appear to have resisted formulation since at least the 1950's. Several relations have, as yet, resisted formulation. This may be due to the practical fact that cohomology algebras over the Steenrod algebra have been a more versatile tool for calculation and a 
richer source of study and have possibly eclipsed study of integral operations. The examples of this article have shown that a knowledge of relations on integral cohomology operations is beneficial to the calculation of the generators of $H^{*}(\mathbb{Z}, n)$ and they have also shown that the integral cohomology algebra is significantly more complex than cohomology algebras with coefficients in finite fields.

\section{Appendix A. The spectral sequence}

The Leray-Serre spectral sequence for a fibration over a simply connected base space $F \rightarrow E \rightarrow B$ is given by

$$
E_{2}^{p, q}=H^{p}\left(B ; H^{q}(F)\right) \Longrightarrow H^{*}(E) .
$$

For the path loop fibration,

$$
K(\mathbb{Z}, n-1) \cong \Omega K(\mathbb{Z}, n) \longrightarrow P K(\mathbb{Z}, n) \longrightarrow K(\mathbb{Z}, n),
$$

where $P K(\mathbb{Z}, n)$ is the path space over $K(\mathbb{Z}, n)$, the sequence converges to the graded cohomology of a contractible space, which tells us that the associated graded algebra is trivial and hence all $E_{\infty}^{p, q}=0$.

This is a spectral sequence of a bigraded differential algebra with the multiplication $E_{n}^{p, q} \times E_{n}^{r, s} \rightarrow E_{n}^{p+r, q+s},(\alpha, \beta) \mapsto \alpha \beta$. As such, for $\alpha \in E_{n}^{p, q}$ and $\beta \in E_{n}^{r, s}$ we have a compatibility condition for the differentials given by

$$
d_{n}(\alpha \beta)=\left(d_{n} \alpha\right) \beta+(-1)^{p+q} \alpha\left(d_{n} \beta\right)
$$

and graded commutativity given by

$$
\alpha \beta=(-1)^{(p+q)(r+s)} \beta \alpha .
$$

In particular $E_{2}^{p, 0} \times E_{2}^{0, s} \rightarrow E_{2}^{p, q}$ is a product $H^{p}(B) \times H^{q}(F) \rightarrow H^{p}\left(B ; H^{q}(F)\right)$.

One form of the universal coefficient theorem yields

$$
\begin{aligned}
H^{p}\left(\mathbb{Z}, n ; H^{q}(\mathbb{Z}, n-1)\right) \cong & H^{q}(\mathbb{Z}, n-1) \otimes H^{p}(\mathbb{Z}, n) \\
& \oplus \operatorname{Tor}\left(H^{q}(\mathbb{Z}, n-1), H^{p+1}(\mathbb{Z}, n)\right) .
\end{aligned}
$$

The term $\operatorname{Tor}\left(H^{q}(\mathbb{Z}, n-1), H^{p+1}(\mathbb{Z}, n)\right)$ will be 0 in all the regions of the spectral sequences that are used in the calculations of this article. We also note the algebraic results $\mathbb{Z} / m\langle\alpha\rangle \otimes \mathbb{Z} / n\langle\beta\rangle \cong \mathbb{Z} / \operatorname{gcd}(m, n)\langle\alpha \otimes \beta\rangle$ and $\mathbb{Z}\langle\gamma\rangle \otimes$ $\mathbb{Z} / n\langle\delta\rangle \cong \mathbb{Z} / n\langle\gamma \otimes \delta\rangle$. Then, in effect, the spectral sequence can be simplified (within the regions used) to

$$
E_{2}^{p, q}=H^{q}(\mathbb{Z}, n-1) \otimes H^{p}(\mathbb{Z}, n) \Longrightarrow 0, p, q \neq 0 \quad \text { and } \quad E_{\infty}^{0,0}=\mathbb{Z} .
$$

The tensor product $\alpha \otimes \beta$ of the generators of $H^{q}(\mathbb{Z}, n-1)$ and $H^{p}(\mathbb{Z}, n)$ that generates $H^{p}\left(\mathbb{Z}, n ; H^{q}(\mathbb{Z}, n-1)\right)$ is identified with the spectral sequence multiplication and denoted $\alpha \beta$.

In the sub-algebras $E_{2}^{p, 0}, 0 \leq p$ and $E_{2}^{0, q}, 0 \leq q$ the spectral sequence multiplications are the cup products in the coП-algebras of $H^{*}(\mathbb{Z}, n)$ and $H^{*}(\mathbb{Z}, n-1)$ respectively ([6], Proposition 5.5). 


\section{Appendix B. Calculation of $H^{m}(\mathbb{Z}, 3)$ for $m \leq 13$}

To show that $H^{*}(\mathbb{Z}, 2) \cong \mathbb{Z}[a]$ we could use the Leray-Serre spectral sequence as we have set it up for the fibration

$$
K(\mathbb{Z}, 1) \cong S^{1} \longrightarrow P \mathbb{C} P^{\infty} \longrightarrow C P^{\infty} \cong K(\mathbb{Z}, 2) .
$$

Since $S^{1}$ has only two non-trivial cohomology groups $H^{0}\left(S^{1}\right) \cong \mathbb{Z}\langle 1\rangle$, the coefficient group generated by 1 , and $H^{1}\left(S^{1}\right) \cong \mathbb{Z}\langle y\rangle$ generated by $y$ (which can be taken as the identity map $S^{1} \rightarrow S^{1}$ ) the calculation is quite simple and we proceed to the calculation of $H^{m}(\mathbb{Z}, 3)$.

The fibration of interest is $K(\mathbb{Z}, 2) \rightarrow P K(\mathbb{Z}, 3) \rightarrow K(\mathbb{Z}, 3)$. From stability we know $H^{3}(\mathbb{Z}, 3) \cong H^{2}(\mathbb{Z}, 2)$ so that $H^{3}(\mathbb{Z}, 3) \cong \mathbb{Z}\langle b\rangle$, where the generator is the transgression $b=\Omega^{-1} a$. Then the $E_{2}$ page remains unchanged into the $E_{3}$ page which begins:

$$
\begin{array}{ccccccc}
\mathbb{Z}\left\langle a^{2}\right\rangle & 0 & 0 & \mathbb{Z}\left\langle a^{2} b\right\rangle & & & \\
0 & 0 & 0 & 0 & 0 & 0 & 0 \\
\mathbb{Z}\langle a\rangle & 0 & 0 & \mathbb{Z}\langle a b\rangle & & & \\
0 & 0 & 0 & 0 & 0 & 0 & 0 \\
\mathbb{Z}\langle 1\rangle & 0 & 0 & \mathbb{Z}\langle b\rangle & ? & ? & ?
\end{array}
$$

We gain some knowledge of the differential since $0 \rightarrow \mathbb{Z}\langle a\rangle \rightarrow \mathbb{Z}\langle b\rangle \rightarrow 0$ must be exact, otherwise $E_{4}^{0,2}$ and $E_{4}^{3,0}$ would survive to $E_{\infty}$, so $d_{3}(a)=b$. We see that $E_{3}^{4,0} \cong E_{\infty}^{4,0} \cong 0$ therefore $H^{4}(\mathbb{Z}, 3) \cong 0$. We also have a sequence

$$
0 \longrightarrow \mathbb{Z}\left\langle a^{2}\right\rangle \longrightarrow \mathbb{Z}\langle a b\rangle \longrightarrow E_{3}^{6,0} \longrightarrow 0
$$

which must be exact at $E_{3}^{3,2}$ and $E_{3}^{6,0}$ since each of these groups must vanish on the $E_{4}$ page or survive to $E_{\infty}$. We have

$$
\begin{aligned}
& d_{3}\left(a^{2}\right)=\left(d_{3} a\right) a+(-1)^{4} a\left(d_{3} a\right)=b a+a b=(-1)^{(4)(3)} a b+a b=2(a b), \\
& d_{3}(a b)=\left(d_{3} a\right) b+(-1)^{2} a\left(d_{3} b\right)=b^{2} \quad(=b \cup b)
\end{aligned}
$$

so that $E_{3}^{6,0}=H^{6}(\mathbb{Z}, 3) \cong \mathbb{Z} / 2\left\langle b^{2}\right\rangle$.

From the above sequence $E_{4}^{0,4}=0$ and hence, on the $E_{4}$ page, $E_{4}^{5,0}=E_{\infty}^{5,0} \cong$ 0 so $H^{5}(\mathbb{Z}, 3) \cong 0$. $E_{3}^{6,2}=\mathbb{Z}\langle a\rangle \otimes \mathbb{Z} / 2\left\langle b^{2}\right\rangle \cong \mathbb{Z} / 2\left\langle a b^{2}\right\rangle$ and we next consider the $E_{3}$ sequence

$$
0 \longrightarrow \mathbb{Z}\left\langle a^{3}\right\rangle \longrightarrow \mathbb{Z}\left\langle a^{2} b\right\rangle \longrightarrow \mathbb{Z} / 2\left\langle a b^{2}\right\rangle \longrightarrow E_{3}^{9,0} \longrightarrow 0 .
$$

We have

$$
\begin{aligned}
d_{3}\left(a^{3}\right) & =\left(d_{3} a^{2}\right) a+(-1)^{4} a^{2}\left(d_{3} a\right)=(-1)^{(2)(3)} 2 a^{2} b+a^{2} b=3\left(a^{2} b\right), \\
d_{3}\left(a^{2} b\right) & =\left(d_{3} a^{2}\right) b+(-1)^{4} a^{2}\left(d_{3} b\right)=2\left(a b^{2}\right)=0\left(\text { since } a b^{2} \text { has order } 2\right), \\
d_{3}\left(a b^{2}\right) & =\left(d_{3} a\right) b^{2}+(-1)^{4} a\left(d_{3} b^{2}\right)=b^{3} .
\end{aligned}
$$


We see that $E_{4}^{0,6} \cong 0$ since $3 a^{2} b$ has infinite order so ker $d_{3}=0$. This means that $E_{3}^{7,0}=E_{\infty}^{7,0}=0$ and hence $H^{7}(\mathbb{Z}, 3)=0$.

$E_{4}^{3,4}=\mathbb{Z} / 3$ and hence on the $E_{5}$ page we get a sequence $0 \rightarrow \mathbb{Z} / 3 \rightarrow$ $E_{5}^{8,0} \rightarrow 0$ which must be exact. Hence $H^{8}(\mathbb{Z}, 3) \cong \mathbb{Z} / 3\langle c\rangle$, where the generator $c$ is indecomposable since it is not given as a cup product by the algebraic structure of the spectral sequence and also the composition $x \circ b^{2}=0$ since $x \in H^{8}(\mathbb{Z}, 6) \cong H^{5}(\mathbb{Z}, 3) \cong 0$ by stability. There is no cross-cap product possible in $H^{8}(\mathbb{Z}, 3)$ for dimensional (degree of the groups involved) reasons (see Section 5).

We will shortly consider the next long sequence (7) in which we will show that $E_{4}^{0,8} \cong 0$ and this means that $E_{4}^{9,0} \cong E_{\infty}^{9,0} \cong 0$ so (6) must be exact at $E_{3}^{9,0}$. Since $d_{3}\left(a^{2} b\right)=0$ we effectively have $0 \rightarrow \mathbb{Z} / 2 \rightarrow E_{3}^{9,0} \rightarrow 0$ so that $H^{9}(\mathbb{Z}, 3) \cong \mathbb{Z} / 2\left\langle b^{3}\right\rangle$.

We now have more information that we can record on the $E_{3}$ page.

$$
\begin{aligned}
& \mathbb{Z}\left\langle a^{4}\right\rangle \quad 0 \quad 0 \quad \mathbb{Z}\left\langle a^{4} b\right\rangle \quad 0 \quad 0 \\
& \begin{array}{lllllll}
0 & 0 & 0 & 0 & 0 & 0 & 0
\end{array} \\
& \mathbb{Z}\left\langle a^{3}\right\rangle \quad 0 \quad 0 \quad 0 \quad \mathbb{Z}\left\langle a^{3} b\right\rangle \quad 0 \quad 0 \quad 0 \quad \mathbb{Z} / 2\left\langle a^{3} b^{2}\right\rangle \\
& \begin{array}{lllllllllll}
0 & 0 & 0 & 0 & 0 & 0 & 0 & 0 & 0 & 0 & 0
\end{array} \\
& \mathbb{Z}\left\langle a^{2}\right\rangle \quad 0 \quad 0 \quad 0 \quad \mathbb{Z}\left\langle a^{2} b\right\rangle \quad 0 \quad 0 \quad 0 \quad \mathbb{Z} / 2\left\langle a^{2} b^{2}\right\rangle \quad 0 \quad \mathbb{Z} / 3\left\langle a^{2} c\right\rangle \quad \mathbb{Z} / 2\left\langle a^{2} b^{3}\right\rangle \\
& \begin{array}{lllllllllllll}
0 & 0 & 0 & 0 & 0 & 0 & 0 & 0 & 0 & 0 & 0 & 0 & 0
\end{array} \\
& \mathbb{Z}\langle a\rangle \quad 0 \quad 0 \quad 0 \quad \mathbb{Z}\langle a b\rangle \quad 0 \quad 0 \quad \mathbb{Z}^{2} / 2\left\langle a b^{2}\right\rangle \quad 0 \quad \mathbb{Z}^{2} / 3\langle a c\rangle \quad \mathbb{Z} / 2\left\langle a b^{3}\right\rangle
\end{aligned}
$$

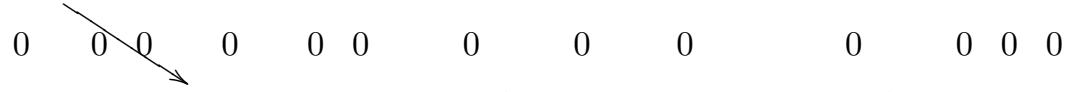

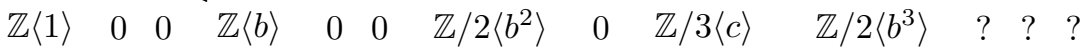

We consider the following $E_{3}$ sequence

$$
0 \longrightarrow \mathbb{Z}\left\langle a^{4}\right\rangle \longrightarrow \mathbb{Z}\left\langle a^{3} b\right\rangle \longrightarrow \mathbb{Z} / 2\left\langle a^{2} b^{2}\right\rangle \longrightarrow \mathbb{Z} / 2\left\langle a b^{3}\right\rangle \longrightarrow E_{3}^{12,0} \longrightarrow 0 .
$$

We have

$$
\begin{aligned}
& d_{3}\left(a^{4}\right)=4\left(a^{3} b\right), \quad \quad d_{3}\left(a^{3} b\right)=3\left(a^{2} b^{2}\right)=a^{2} b^{2}, \\
& d_{3}\left(a^{2} b^{2}\right)=2\left(a b^{3}\right)=0, \quad d_{3}\left(a b^{3}\right)=b^{4},
\end{aligned}
$$

from which we get $E_{4}^{0,8} \cong 0, E_{4}^{3,6} \cong\left\langle 2\left(a^{3} b\right)\right\rangle /\left\langle 4\left(a^{3} b\right)\right\rangle \cong \mathbb{Z} / 2$ and $E_{4}^{6,4} \cong 0$. Since $d_{3}\left(a^{2} b^{2}\right)=0$ we effectively have $0 \rightarrow E_{3}^{9,2} \rightarrow E_{3}^{12,0} \rightarrow 0$. $E_{4}^{9,2}=E_{\infty}^{9,2}$, since we will see in (9) that $E_{4}^{0,10} \cong 0$ so exactness at $E_{3}^{9,2}$ means that $E_{3}^{12,0}$ must have a summand $\mathbb{Z} / 2\left\langle b^{4}\right\rangle$. On the $E_{3}$ page we also have the sequence

$$
0 \longrightarrow \mathbb{Z} / 3\langle a c\rangle \longrightarrow E_{3}^{11,0} \longrightarrow 0
$$


and since $E_{4}^{6,4} \cong 0$ and $E_{4}^{0,10} \cong 0, E_{4}^{11,0}=E_{\infty}^{11,0}$ and (8) must be exact at $E_{3}^{11,0}$. Since $d_{3}(a c)=b c$ we must consider two cases depending on whether $E_{3}^{11,0} \cong 0$ or $¥ 0$.

If $E_{3}^{11,0} \cong 0$, then $d_{3}(a c)=0$ so $E_{4}^{8,2}=\mathbb{Z} / 3$ hence $0 \rightarrow E_{5}^{3,6} \rightarrow E_{5}^{8,2} \rightarrow 0$ must be exact at $E_{5}^{8,2}$ since $E_{6}^{8,2}=E_{\infty}^{8,2} \cong 0$. However, since there can only be the trivial homomorphism $d_{5}: \mathbb{Z} / 2 \rightarrow \mathbb{Z} / 3$ we cannot have exactness at $E_{5}^{8,2}$ and this contradiction leads us to conclude that $E_{3}^{11,0} \neq 0$.

With $E_{3}^{11,0} \approx 0$ we get $d_{3}(a c)=b c$ generates $E_{3}^{11,0}$ so $H^{11}(\mathbb{Z}, 3) \cong \mathbb{Z} / 3\langle b c\rangle$. Then $E_{4}^{8,2} \cong 0$ and the exact sequence $0 \rightarrow \mathbb{Z} / 2 \rightarrow E_{7}^{10,0} \rightarrow 0$ yields $H^{10}(\mathbb{Z}, 3) \cong$ $\mathbb{Z} / 2\langle d\rangle$. At this stage we cannot determine whether the generator $d$ is indecomposable, it could, for instance, be given by $x \circ b^{2}$ with $x \in H^{10}(\mathbb{Z}, 6)$. We discussed this in Section 4.

Returning to the $E_{3}$ page we have sequences

$$
\begin{gathered}
0 \longrightarrow \mathbb{Z}\left\langle a^{5}\right\rangle \longrightarrow \mathbb{Z}\left\langle a^{4} b\right\rangle \longrightarrow \mathbb{Z} / 2\left\langle a^{3} b^{2}\right\rangle \longrightarrow \mathbb{Z} / 2\left\langle a^{2} b^{3}\right\rangle \\
\longrightarrow \mathbb{Z} / 2\left\langle a b^{4}\right\rangle \oplus ? \longrightarrow E_{3}^{15,0} \longrightarrow 0, \\
0 \longrightarrow \mathbb{Z} / 3\left\langle a^{2} c\right\rangle \longrightarrow \mathbb{Z} / 3\langle a b c\rangle \longrightarrow E_{3}^{14,0} \longrightarrow 0, \\
0 \longrightarrow \mathbb{Z} / 2\langle a d\rangle \longrightarrow E_{3}^{13,0} \longrightarrow 0 .
\end{gathered}
$$

From (9) we calculate $E_{4}^{0,10} \cong 0, E_{4}^{3,8} \cong \mathbb{Z} / 5, E_{4}^{6,6} \cong 0$ and $E_{4}^{9,4} \cong 0$

From (10) we get $E_{4}^{8,4} \cong 0$. Then on the $E_{4}$ page we see $E_{4}^{11,2}=E_{\infty}^{11,2} \cong 0$ so that (10) must be exact at $E_{3}^{11,2}$. Since $d_{3}\left(a^{2} c\right)=2(a b c)$ generates $E_{3}^{11,2}$ we must also have ker $d_{3}=E_{3}^{11,2}$ and hence $d_{3}(a b c)=b^{2} c=0$. This tells us that $H^{14}(\mathbb{Z}, 3)$ does not have a non-trivial summand generated by the cup product $b \cup b \cup c$. We discussed this result in Section 5 .

From (11) since $E_{4}^{13,0}=E_{\infty}^{13,0} \cong 0$ we have exactness at $E_{3}^{13,0}$. Again, two cases arise $E_{3}^{13,0} \cong 0$ or $¥ 0$. The first case leads to a contradiction since we then have $E_{3}^{10,2}=E_{4}^{10,2} \cong \mathbb{Z} / 2$ which leads to $d_{7}: \mathbb{Z} / 5 \rightarrow \mathbb{Z} / 2$, which can only be trivial, resulting in $E_{8}^{10,2}=E_{\infty}^{10,2} \neq 0$. Hence $d_{3}(a d)=b d$ generates $E_{3}^{13,0}$ and we have $H^{13}(\mathbb{Z}, 3) \cong \mathbb{Z} / 2\langle b d\rangle$.

On the $E_{9}$ page we get a sequence

$$
0 \longrightarrow \mathbb{Z} / 5 \longrightarrow E_{9}^{12,0} \longrightarrow 0
$$

which must be exact everywhere, hence $E_{9}^{12,0} \cong \mathbb{Z} / 5\langle e\rangle$ and the complete calculation gives $H^{12}(\mathbb{Z}, 3) \cong \mathbb{Z} / 2\left\langle b^{4}\right\rangle \oplus \mathbb{Z} / 5\langle e\rangle$, with the generator $e$ undetermined, as decomposable or not, at this time.

Further partial results can be obtained; in some instances the uncertainties that arise for the differentials can not be resolved on a later page because they do not lead to contradictions. Where the uncertainty of the differentials leads to groups of different torsion, knowledge of the modulo $p$ cohomology, as given in [1], may be sufficient to resolve the problem. In this paper we were content to pass to the cohomology of $K(\mathbb{Z}, 4)$ at this stage. 
Proceeding in a similar way we take the spectral sequence for the fibration

$$
K(\mathbb{Z}, 3) \longrightarrow P K(\mathbb{Z}, 4) \longrightarrow K(\mathbb{Z}, 4)
$$

and having calculated the groups $H^{m}(\mathbb{Z}, 4)$ as far as possible we can then look at the spectral sequence for

$$
K(\mathbb{Z}, 4) \longrightarrow P K(\mathbb{Z}, 5) \longrightarrow K(\mathbb{Z}, 5)
$$

and so on. For the calculation of $H^{13}(\mathbb{Z}, 4)$ we use the fact, determined on the $E_{7}$ page, that $d_{7}\left(b^{2}\right)=g$ together with the derivation property.

\section{References}

[1] H. Cartan, Sur les groupes d'Eilenberg-Mac Lane. II, Proc. Nat. Acad. Sci. U. S. A. 40 (1954), 704-707.

[2] W. Dreckmann, On the definition of П-algebras, C. R. Acad. Sci. Paris Ser. I Math. 321 (1995), no. 6, 767-772.

[3] S. Eilenberg and S. MacLane, On the groups $H(\Pi, n)$. II, Methods of computation. Ann. of Math. (2) 60, (1954), 49-139.

[4] S. O. Kochman, Integral cohomology operations, Current trends in algebraic topology, Part 1 (London, Ont., 1981), pp. 437-478, CMS Conf. Proc., 2, Amer. Math. Soc., Providence, R.I., 1982.

[5] J. P. May, A general algebraic approach to Steenrod operations, The Steenrod Algebra and its Applications (Proc. Conf. to Celebrate N. E. Steenrod's Sixtieth Birthday, Battelle Memorial Inst., Columbus, Ohio, 1970) pp. 153-231, Lecture Notes in Mathematics, Vol. 168 Springer, Berlin, 1970.

[6] J. McCleary, User's Guide To Spectral Sequences, Mathematics Lecture Series, 12. Publish or Perish, Inc., Wilmington, DE, 1985.

[7] A. Percy, An Eckmann-Hilton dual to the П-algebras of homotopy theory, Illinois J. Math. 48 (2004), no. 4, 1305-1320.

[8] N. Steenrod, Cohomology operations, and obstructions to extending continuous functions, Advances in Math. 8 (1972), 371-416.

[9] H. Toda, Composition Methods in Homotopy Groups of Spheres, Annals of Mathematics Studies, No. 49 Princeton University Press, Princeton, N.J. 1962.

School of Applied Science and Engineering

Monash University

Churchill, VIC 3842, Australia

E-mail address: andrew.percy@sci.monash.edu.au 